EDITOR'S

EDITOR'S

CHOICE

\title{
Urine catecholamines in paediatrics
}

Daniel J Erdelyi, Martin Elliott, Bob Phillips

- A supplementary table is published online only. To view this file please visit the journal online (http://adc.bmj.com)

Regional Department of Paediatric Haematology and Oncology, Leeds Children's Hospital, Leeds, UK

\section{Correspondence to}

Dr Daniel J Erdelyi, Regional Department of Paediatric

Haematology and Oncology, Leeds Children's Hospital,

Clarendon Road, Leeds LS1 3EX, UK;

erddan@gmail.com

Accepted 9 March 2011

\section{INTRODUCTION}

In paediatric clinical practice, tests for urinary catecholamines and their metabolites are most often requested to aid in the diagnosis and follow-up of patients with neural crest tumours, particularly neuroblastomas.

Neuroblastoma is the most common extracranial solid tumour in children. Each year 80-90 new patients are diagnosed in the UK, most of them infants or toddlers. Neuroblastoma usually arises from either the adrenal medulla or the paravertebral sympathetic chain. It shows a wide variety of biological activity and has a correspondingly diverse range of presenting features and subsequent prognoses. Younger patients below 18 months of age generally have less aggressive forms of the tumour and require less intensive treatment, and indeed some children may not require any treatment as the tumour can spontaneously remit. In contrast, older children often present with more advanced and biologically aggressive forms of the disease and outcome is often poor despite very intensive treatment.

The presenting symptoms are usually dictated by the site of the primary tumour, sites of metastatic disease (bone marrow, bone, lymph nodes, liver, skin, brain and lungs) and possible hormone secretion. A suspicion of neuroblastoma is most typically raised in a child found to have abdominal, mediastinal or bone masses, or in a child with non-specific symptoms of lethargy, weight loss, recurrent infections or fever. Neuroblastoma should also be considered in any child with Horner's syndrome, opsoclonus-myoclonus syndrome or a persistent limp.

Phaeochromocytoma is another tumour which secretes catecholamines and can be either benign or malignant. These tumours are extremely rare in childhood, with only one or two new cases diagnosed in the UK each year, usually in teenagers. Presenting symptoms include sweating, palpitations, syncope, tremor and weight loss, which are all related to catecholamine secretion, although occasionally patients present with mass effect from the tumour. These patients often have hypertension, which can be paroxysmal.

Localising symptoms warrant urgent imaging, but in all other cases, especially if symptoms are non-specific, a simple urine test with rule-in or rule-out potential would be a valuable diagnostic tool and aid further specialist referral. In this article we discuss the practicalities of a urine catecholamine test and available evidence regarding the utility of such a test in diagnosing neuroblastoma and phaeochromocytoma, and in following up patients after treatment is completed. Further information and critical appraisal of the papers cited in relation to the specific clinical questions can be found in the supplementary online table.

\section{PHYSIOLOGICAL BACKGROUND}

The physiological, and hence pathophysiological, basis for measuring urinary catecholamines and metabolites relies on a simple understanding of the metabolic pathways involved. Endogenous catecholamines act as neurotransmitters in the central nervous system and the sympathetic postganglionic endings and are secreted as hormones by the chromaffin cells of the adrenal medulla. They are synthesised from tyrosine and stored in special granules in the producing cells. After release, their half-life in the plasma is about $1 \mathrm{~min}$. Only $2-3 \%$ of catecholamines are excreted unchanged in the urine, with most of them being metabolised by various enzymatic pathways into many metabolites, which can be detected in urine. ${ }^{1}$ The main components of the metabolic pathways and, in particular, the chemicals measured as part of the urine catecholamines are detailed in figure 1.

The adrenal medullae secrete epinephrine and to a lesser degree norepinephrine, while pure norepinephrine is released from sympathetic postganglionic endings, and collectively these are important in physiological acute stress reactions. Their biosynthesis and release is regulated dominantly by cholinergic sympathetic innervation. Their effects include tachycardia, increased myocardial contractility, mobilisation of glucose and fatty acids from stores and increased metabolic rate, dilatation of arterioles in muscles but constriction in viscera and skin, and smooth muscle relaxation in bronchi, bowels and the bladder. Normal levels of plasma and urine catecholamines are very variable and change in response to posture, stress, pain, plasma glucose level, and so on. ${ }^{2}$ Drugs and in particular, aminophylline, caffeine, erythromycin, salicylates, monoamine oxidase inhibitors and imipramine can have major effects on catecholamine levels.

Neuroblastomas usually have high expression of tyrosine hydroxylase, the enzyme which converts tyrosine to L-dopa, which is then metabolised to dopamine and homovanillic acid (HVA; figure 1). Some of the dopamine is converted to norepinephrine and epinephrine and these are then metabolised to vanillylmandelic acid (VMA).

Phaeochromocytomas tend to produce more epinephrine and norepinephrine and the metabolite VMA, relative to dopamine and HVA. This pattern of catecholamine secretion may also be seen in infants with neuroblastoma. Regardless of the underlying tumour type, it is extremely important that patients with this pattern of catecholamine secretion have appropriate $\alpha$-blockade 
prior to any surgical intervention as surgical manipulation of these tumours can produce acute catecholamine release and catastrophic changes in blood pressure.

\section{TECHNICALITIES OF TESTING}

Unless the specimen is processed within a few hours of urination, the sample should be acidified to $\mathrm{pH} 4$ using reagents to prevent degradation of catecholamines. In order to avoid acid burns, it is advisable that the patient does not pass urine directly into an acid-containing bottle, but that reagents are added afterwards. In the case of a $24 \mathrm{~h}$ collection, passed samples can be poured one by one into a bottle containing the reagents. ${ }^{3}$

Urine catecholamines and their metabolites are most commonly analysed using high performance liquid chromatography (HPLC), although other techniques including mass spectrometry and immunoassay are sometimes used. Before HPLC can be performed, catecholamines are extracted from the urine and the purified extract is then injected onto an HPLC column. Analytes elute from the column at known retention times, and pass into an electrochemical detector where they are oxidised and each compound appears as a chromatographic peak, the area of which is directly related to concentration. The concentrations are then expressed as either $\mu \mathrm{mol} / 24 \mathrm{~h}$, or more commonly in children, $\mu \mathrm{mol} / \mathrm{mmol}$ creatinine. An added reference compound is also measured to correct the process for any variations in extraction efficacy and elution between analyses. ${ }^{1}$

Each laboratory creates age-adjusted normal ranges of urine catecholamines and metabolites as determined by their own methodology and use of specific reagents, by testing urine from patients who do not have a catecholamine producing tumour. As a result, the normal ranges quoted vary among different laboratories.<smiles>NC(Cc1ccc(O)c(O)c1)C(=O)O</smiles>
DOPA
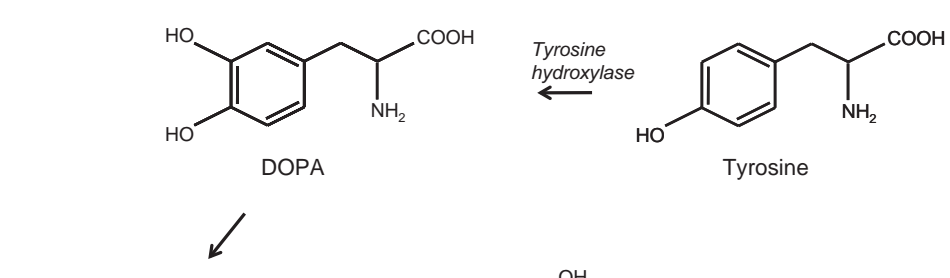<smiles>NCCc1ccc(O)c(O)c1</smiles>

Dopamine
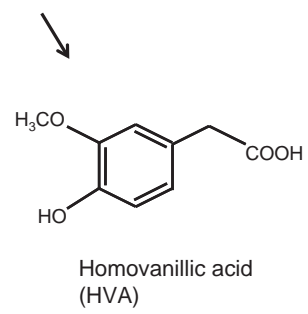

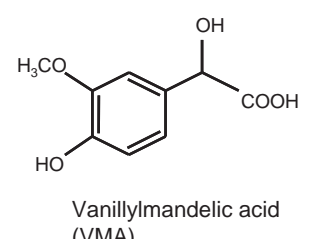

(VMA)
Figure 1 Simplified pathway of catecholamine synthesis and metabolism.

\section{CLINICAL QUESTIONS}

\section{Can we use the test to diagnose neuroblastoma?}

In a child with suspected neuroblastoma [patient] do urinary catecholamine metabolites [test] accurately diagnose neuroblastoma [outcome]? Which metabolites have the greatest diagnostic value?

One of the challenges when investigating a patient with suspected neuroblastoma is establishing a definitive diagnosis using minimally invasive procedures in a timely manner. Knowing the probable diagnosis before biopsy is useful in decisions regarding mode of biopsy, the concurrent placement of a central line for chemotherapy, and the exclusion of conditions requiring quite different treatment modalities.

Urine catecholamines have been used since the early 1970s to assist in the diagnosis of neuroblastoma. ${ }^{4}$ The most effective, regularly assessed, catecholamine metabolites are HVA and VMA. There are some suggestions that methylated variants may have marginally greater diagnostic value. ${ }^{56}$ Various studies ${ }^{4-15}$ have shown that abnormal values have a sensitivity of $66-100 \%$. The proportion of neuroblastoma patients with elevated urinary levels of VMA and HVA varies with the stage of the disease (as described by the International Neuroblastoma Staging System), with low stage tumours being less likely to have abnormal levels (see table 1).

Specificity is generally very high, with estimates of $>99 \%$ and in one series $5100 \%$ specificity was reported. False positive results are occasionally found, and these often seem to occur in children with other chronic health disorders, such as severe eczema, muscular dystrophy and congestive cardiac failure. ${ }^{7}$ Most series report a positive result when the level is at least 1.5 times the upper limit of normal and results which are much higher than the age-related upper limit of normal are increasingly likely to represent true positives.

Not surprisingly, the chance of a urine catecholamine test being positive depends on the clinical context, that is, a positive result is very unlikely in a screening programme, but highly likely in an ill child with an adrenal mass. The relative probability of children in differing clinical scenarios having neuroblastoma dependent on urine catecholamine results is shown in table 2.

\section{Clinical bottom lines}

1. In patients with a moderate suspicion of neuroblastoma, a positive urine catecholamine test

Table 1 Sensitivity of abnormal homovanillic acid (HVA) or vanillylmandelic acid (VMA) results in relation to the International Neuroblastoma Staging System stage of neuroblastoma 8121315

\begin{tabular}{ll}
\hline Stage of disease & $\begin{array}{l}\text { Sensitivity of abnormal } \\
\text { HVA and/or VMA }\end{array}$ \\
\hline 1 & $78-85 \%$ \\
$2-3$ & $80-100 \%$ \\
4 & $92-100 \%$ \\
$4 \mathrm{~S}$ & $100 \%$ \\
\hline
\end{tabular}


Table 2 Probability of patients in different clinical contexts having neuroblastoma based on positive and negative urine catecholamine and metabolite tests

\begin{tabular}{lclc}
\hline Population & $\begin{array}{l}\text { Pretest (background) } \\
\text { probability of } \\
\text { neuroblastoma }\end{array}$ & Test result & $\begin{array}{l}\text { Post-test } \\
\text { probability of } \\
\text { neuroblastoma }\end{array}$ \\
\hline $\begin{array}{l}\text { Vague suspicion of neuroblastoma, } \\
\text { non-specifically unwell child }\end{array}$ & $2 \%$ & Positive & $63 \%$ \\
$\begin{array}{l}\text { Secondary care, distinct suspicion of } \\
\text { neuroblastoma }\end{array}$ & $10 \%$ & Negative & $0 \%$ \\
$\begin{array}{l}\text { Paraspinal or renal mass, strongly } \\
\text { suspected neuroblastoma }\end{array}$ & $60 \%$ & Positive & $99 \%$ \\
Unilateral Horner's syndrome ${ }^{14}$ & $8 \%$ & Negative & $1 \%$ \\
Well infants $~ 6$ months old, screen- & $0.05 \%$ & Positive & $99.9 \%$ \\
ing investigation & & Negative & $18 \%$ \\
Opsoclonus-myoclonus syndrome & & Positive & $99 \%$ \\
& $53 \%$ & Negative & $1 \%$ \\
\hline
\end{tabular}

Assumes average likelihood ratios (LR) for abnormal urine catecholamines of $L R+85, L R-0.15$ (sensitivity $\sim 85 \%$ and specificity $\sim 99 \%$ ).

warrants further diagnostic evaluation, including repeat urine testing and abdominal and thoracic imaging.

2. In patients who are non-specifically unwell, with only a moderate suspicion of neuroblastoma, a negative urine result is likely to rule out the diagnosis.

3. In patients with a strong suspicion of neuroblastoma in whom the test is negative, the diagnosis has not been fully excluded and further evaluation is still required.

\section{Do I need to do $24 \mathrm{~h}$ collection or is a single, spot sample good enough?}

In a child with suspected neuroblastoma [patient] do random or untimed urinary catecholamine metabolites [test] have a similar diagnostic value [outcome] to $24 \mathrm{~h}$ collections [comparison]?

Logistically a $24 \mathrm{~h}$ collection on an infant or young child is difficult and unlikely to be a complete collection. Some work in countries with neuroblastoma screening policies has shown diurnal variation in metabolite excretion, ${ }^{12}$ and this has been well documented in the setting of phaeochromocytoma. ${ }^{16}$ Very few direct comparisons of methods have been reported, however an indirect assessment can be carried out by reviewing the published literature and comparing the sensitivity of the tests used.

Direct comparisons show excellent correlation between the results of $24 \mathrm{~h}$ and random samples using different laboratory techniques, ${ }^{17}$ and the lack of clinical importance of $24 \mathrm{~h}$ samples is emphasised by the observation that negative spot samples are also negative on 24 h collections. ${ }^{18}$ Further indirect evidence demonstrates that $24 \mathrm{~h}$ urine collections from infants are often incomplete and this results in a high frequency of inaccurate results. ${ }^{5} 12$ In addition, large trials and series used untimed samples in their studies. ${ }^{8} 19$

Despite the physiological concern that diurnal variation may create false negative results, fortunately for clinicians and patients, evidence suggests that untimed or random specimens are likely to be as effective for making a diagnosis of neuroblastoma as a $24 \mathrm{~h}$ collection.

\section{Clinical bottom line}

1. Untimed, also known as random or spot, urine collection methods to determine urinary catecholamines are as effective as $24 \mathrm{~h}$ collections and far more practical.

\section{Should I use the test to follow-up patients who have completed treatment for neuroblastoma?}

In a child who has completed treatment for neuroblastoma [patient] do urinary catecholamine metabolites [test] accurately detect relapse [outcome]? Does this vary by initial secreting status of the tumour?

There is no clear answer to the question of how valuable urinary catecholamines are in the detection of relapsing neuroblastoma and it probably depends on which other investigations are being used to identify patients with relapsing disease. The specificity of the test in this context has not been reported, but extrapolating from reports of the initial diagnostic value of catecholamine metabolites would suggest that the test is highly specific and there is no reason why the specificity would be any different in patients with relapsing neuroblastoma compared to those at initial diagnosis. Most reports do not mention having a lower threshold for urine catecholamine levels which would prompt further investigation to detect relapse in patients on follow-up surveillance, compared to the usual two or three times the upper limit of normal used in the primary diagnosis of neuroblastoma. Practically, most patients with an elevated level of urine catecholamines on two successive samples would progress to have further investigations.

There are a range of values for test sensitivity in detecting relapse. One report from the USA shows very poor sensitivity (24\%) in 154 patients with relapsed disease when compared to regular radionucleotide imaging (metaiodobenzylguanidine, MIBG scans) in the highest risk group of patients with stage 4 neuroblastoma. ${ }^{20}$ This would suggest that reliance on urine catecholamine testing would result in a lag period in detecting relapse compared to using more invasive investigations. A small Japanese study ${ }^{21}$ suggested that sensitivity is around $45 \%$, but this had only 11 relapse episodes in eight patients, and these patients had mixed stages of neuroblastoma. A larger European study $^{22}$ showed a similar sensitivity of $54 \%$ (47 out of 87 patients with relapse or progression), which was much lower than with MIBG imaging which had a sensitivity of $94 \%$ in a similar group of patients. This group used a less intensive radiological surveillance strategy, which may reflect the higher sensitivity of the tests compared to the US study which was identifying relapsed patients at an earlier time point. Taken together, these data suggest that urine catecholamine metabolites become abnormal in roughly the same proportion 
of patients as at initial diagnosis. Other techniques probably can detect relapse earlier, but they are more invasive.

The value of early detection of neuroblastoma relapse in patients without symptoms is debatable. In particular, patients with relapsed high risk neuroblastoma have a very poor prognosis and are unlikely to be cured, although treatment may be valuable in symptomatic palliation. The treatment of relapsed disease is subject to ongoing study.

\section{Clinical bottom line}

1. Patients with relapsed disease will have elevated urine catecholamines and metabolites as at initial diagnosis, although other modalities of surveillance, particularly MIBG-radionucleotide imaging, may detect relapsed disease earlier compared to urine catecholamine testing alone.

Can I use the test to rule out phaeochromocytoma? In a child with hypertension [patient] do urinary catecholamine metabolites [test] accurately diagnose phaeochromocytoma [outcome]? Which metabolites have the greatest diagnostic value?

Phaeochromocytoma is an exceptionally rare diagnosis in children as discussed in the

\section{QUIZ}

\section{QUESTION 1}

Which of the statements below are true?

A. Norepinephrine is excreted as a neurotransmitter from the parasympathetic preganglionic neural endings.

B. Norepinephrine is excreted as a neurotransmitter in the central nervous system.

C. Urine catecholamine levels and their metabolites are usually measured using high performance liquid chromatography.

D. A high urine vanillylmandelic acid/homovanillic acid ratio is suggestive of high epinephrine and norepinephrine secretion by the tumour.

E. Phaeochromocytoma is more common than neuroblastoma among children.

\section{QUESTION 2}

In which clinical situations would normal urine catecholamine results rule out a diagnosis of neuroblastoma with considerable reliability?
A. Opsoclonus-myoclonus syndrome
B. A 3-week history of weight loss, fatigue and low grade fevers
C. Horner's triad
D. Thoracic paraspinal mass

\section{QUESTION 3}

Which of the statements below are true?

A. In a child with isolated hypertension, testing urine catecholamines is of little value for diagnosing or excluding phaeochromocytoma.

B. Isotope scans usually detect neuroblastoma relapse earlier than urine catecholamine testing.

C. Urine catecholamine testing is an effective screening tool for infantile neuroblastoma and this is expected to become standard in the UK in the future.

D. Physiological serum catecholamine levels show diurnal variation.

E. There is evidence that testing catecholamines from a $24 \mathrm{~h}$ urine collection is superior to a random timed single urine sample regarding sensitivity for diagnosing neuroblastoma.

Answers to the quiz are on page 118 introduction. $^{23}$ Various sources agree that it causes less than $1 \%$ of cases of childhood hypertension. ${ }^{24-26}$ The diagnostic accuracy of the test in this setting is estimated to have a sensitivity of $86 \%$ and specificity of $88 \%$; a positive test result has a $3 \%$ chance of phaeochromocytoma, and a negative result reduces the probability to $0.01 \% .{ }^{27}$ However, the majority of children with phaeochromocytoma have an underlying inherited genetic predisposition, and in many cases these are syndromes with other obvious manifestations. ${ }^{23}$ Also, in most cases of phaeochromocytoma hypertension is accompanied by other signs, and a higher index of suspicion is warranted especially with the triad of hypertension, sweating and tremors. ${ }^{28}$ Therefore in practice, phaeochromocytoma is almost never the cause of isolateed hypertension. If phaeochromocytoma is strongly suspected, we suggest referral to an endocrine specialist for specialised investigations under close observation for plasma free metanephrines. ${ }^{29}$

\section{Clinical bottom lines}

1. Phaeochromocytoma is an exceptionally rare diagnosis, but if present, other signs of the disease or a predisposing syndrome often accompany hypertension.

2. Without strong clinical suspicion, urine catecholamine testing does not provide useful diagnostic information.

\section{LIMITATIONS}

\section{Use of the test in screening programmes}

Japan has the most experience of neuroblastoma screening programmes and ran a national urine screening programme for all children at the age of 6 months from 1984 to 2003, but this was discontinued as it did not appear to reduce deaths from neuroblastoma; there have been similar results from smaller German and Canadian studies. ${ }^{30} 31$ Screening of patients at 6 or 12 months of age seems to identify patients with a moderate risk of neuroblastoma which in many cases will spontaneously regress. Therefore, some of the patients detected by screening will be treated for a condition which without screening would never have become clinically significant. More recent analysis of the data from Japan comparing those diagnosed in prescreening and postscreening cohorts, however, showed reduced mortality in those diagnosed in the prescreening period, but this difference may be due to therapeutic advances. ${ }^{11}$ Currently there is no clear evidence to suggest that national urine catecholamine testing is beneficial.

\section{Reference ranges}

As explained in the Technicalities of testing section, there is no accepted standard reference range for urine catecholamines and their metabolites, and each laboratory produces their own specific reference ranges. The interpretation of specific values is only meaningful in this context. 


\section{CONCLUSIONS}

Urine catecholamine measurement can be a useful diagnostic test in children with suspected neuroblastoma, but its value depends upon the clinical situation. When clinical signs such as unilateral Horner's syndrome or an abdominal or paraspinal mass result in a high suspicion of a child having neuroblastoma, then a positive test result will essentially make the diagnosis. This does not rule out the value of biopsy, as important diagnostic information which may alter management will be acquired through genetic testing of the tumour material. In the setting of a non-specifically unwell child, a positive test demands further investigation but does not confirm neuroblastoma. These urine tests can be undertaken on a spot sample; a $24 \mathrm{~h}$ collection is unnecessary in this setting. The follow-up of children after completion of therapy can be usefully undertaken with urine catecholamine screening, but this is not the most sensitive method of detecting relapse. Phaeochromocytoma is such a rare diagnosis that even in hypertensive children, a urine catecholamine test is unlikely to suggest a diagnosis and the results may well be unhelpful. Patients strongly suspected of having phaeochromocytoma should be referred for specialist review.

\section{Competing interests None.}

Provenance and peer review Commissioned; externally peer reviewed.

\section{REFERENCES}

1. Tsunoda M. Recent advances in methods for the analysis of catecholamines and their metabolites. Anal Bioanal Chem 2006:386:506-14.

2. Ganong WF. The adrenal medulla and adrenal cortex. In: Ganong WF, ed. Review of Medical Physiology. 2004:270-92.

3. Willemsen JJ, Ross HA, Lenders JW, et al. Stability of urinary fractionated metanephrines and catecholamines during collection, shipment, and storage of samples. Clin Chem 2007:53:268-72

4. LaBrosse EH, Com-Nougué C, Zucker JM, et al. Urinary excretion of 3-methoxy-4-hydroxymandelic acid and 3-methoxy4-hydroxyphenylacetic acid by 288 patients with neuroblastoma and related neural crest tumors. Cancer Res 1980;40:1995-2001.

5. Monsaingeon M, Perel Y, Simonnet G, et al. Comparative values of catecholamines and metabolites for the diagnosis of neuroblastoma. Eur J Pediatr 2003;162:397-402.

6. Candito M, Thyss A, Albertini M, et al. Methylated catecholamine metabolites for diagnosis of neuroblastoma. Med Pediatr Oncol 1992;20:215-20.

7. Bell S, Parker L, Craft AW, et al. False positive results in a neuroblastoma screening programme. Med Pediatr Oncol 1994;22:181-6.

8. Berthold F, Hunneman DH, Harms D, et al. Serum vanillylmandelic acid/homovanillic acid contributes to prognosis estimation in patients with localised but not with metastatic neuroblastoma. Eur J Cancer 1992;28A:1950-4

9. Craft AW, Parker L, Dale G, et al. A pilot study of screening for neuroblastoma in the north of England. Am J Pediatr Hematol Oncol 1992;14:337-41.

10. Graham-Pole J, Salmi T, Anton AH, et al. Tumor and urine catecholamines (CATs) in neurogenic tumors. Correlations with other prognostic factors and survival. Cancer 1983;51:834-9.

11. Hiyama $\mathbf{E}$, lehara $T$, Sugimoto $T$, et al. Effectiveness of screening for neuroblastoma at 6 months of age: a retrospective population-based cohort study. Lancet 2008;371:1173-80.

12. Kellie SJ, Clague AE, McGeary HM, et al. The value of catecholamine metabolite determination on untimed urine collections in the diagnosis of neural crest tumours in children. Aust Paediatr J 1986;22:313-15.

13. Strenger V, Kerbl R, Dornbusch HJ, et al. Diagnostic and prognostic impact of urinary catecholamines in neuroblastoma patients. Pediatr Blood Cancer 2007:48:504-9.

14. Mahoney NR, Liu GT, Menacker SJ, et al. Pediatric horner syndrome: etiologies and roles of imaging and urine studies to detect neuroblastoma and other responsible mass lesions. Am J Ophthalmol 2006;142:651-9.

15. Zambrano E, Reyes-Múgica M. Hormonal activity may predict aggressive behavior in neuroblastoma. Pediatr Dev Pathol 2002;5:190-9.

16. Sullivan JM, Solomon HS. The diagnosis of pheochromocytoma. Overnight excretion of catecholamine metabolites. JAMA 1975;231:618-19.

17. Gregianin LJ, McGill AC, Pinheiro CM, et al. Vanilmandelic acid and homovanillic acid levels in patients with neural crest tumor: 24-hour urine collection versus random sample. Pediatr Hematol Oncol 1997:14:259-65.

18. Tuchman M, Ramnaraine ML, Woods WG, et al. Three years of experience with random urinary homovanillic and vanillylmandelic acid levels in the diagnosis of neuroblastoma. Pediatrics 1987;79:203-5.

19. Ladenstein R, Valteau-Couanet D, Brock P, et al. Randomized Trial of prophylactic granulocyte colony-stimulating factor during rapid COJEC induction in pediatric patients with high-risk neuroblastoma: the European HR-NBL1/SIOPEN study. J Clin Oncol 2010;28:3516-24.

20. Kushner BH, Kramer K, Modak S, et al. Sensitivity of surveillance studies for detecting asymptomatic and unsuspected relapse of high-risk neuroblastoma. J Clin Oncol 2009;27:1041-6.

21. Okuyama C, Ushijima Y, Kubota T, et al. Utility of follow-up studies using meta-[123 I]iodobenzylguanidine scintigraphy for detecting recurrent neuroblastoma. Nucl Med Commun 2002;23:663-72.

22. Simon T, Hero B, Hunneman DH, et al. Tumour markers are poor predictors for relapse or progression in neuroblastoma. Eur J Cancer 2003;39:1899-903.

23. Armstrong R, Sridhar M, Greenhalgh KL, et al. Phaeochromocytoma in children. Arch Dis Child 2008;93:899-904

24. Wyszynska T, Cichocka E, Wieteska-Klimczak A, et al. A single pediatric center experience with 1025 children with hypertension. Acta Paediatr 1992;81:244-6.

25. Grinsell MM, Norwood VF. At the bottom of the differential diagnosis list: unusual causes of pediatric hypertension. Pediatr Nephrol 2009;24:2137-46.

26. McCrindle BW. Assessment and management of hypertension in children and adolescents. Nat Rev Cardiol 2010; 7:155-63

27. Lenders JW, Pacak K, Walther MM, et al. Biochemica diagnosis of pheochromocytoma: which test is best? JAMA 2002:287:1427-34

28. Sullivan J, Groshong T, Tobias JD. Presenting signs and symptoms of pheochromocytoma in pediatric-aged patients. Clin Pediatr (Phila) 2005;44:715-19.

29. Sawka AM, Prebtani AP, Thabane L, et al. A systematic review of the literature examining the diagnostic efficacy of measurement of fractionated plasma free metanephrines in the biochemical diagnosis of pheochromocytoma. BMC Endocr Disord 2004;4:2.

30. Woods WG, Gao RN, Shuster JJ, et al. Screening of infants and mortality due to neuroblastoma. N Engl J Med 2002:346:1041-6.

31. Schilling FH, Spix C, Berthold F, et al. Neuroblastoma screening at one year of age. N Engl J Med 2002;346:1047-53.

32. Stabell N, Nordal E, Stensvold E, et al. Febrile neutropenia in children with cancer: a retrospective Norwegian multicentre study of clinical and microbiological outcome. Scand J Infect Dis 2007:40:301-7.

33. Krug P, Schleiermacher G, Michon J, et al. Opsoclonus myoclonus in children associated or not with neuroblastoma. Eur J Paediatr Neurol 2010;14:400-9.

34. Tate ED, Allison TJ, Pranzatelli MR, et al. Neuroepidemiologic trends in 105 US cases of pediatric opsoclonus-myoclonus syndrome. J Pediatr Oncol Nurs 2005;22:8-19. 